\title{
1 Topical anaesthetic use prior to rigid gas permeable contact lens fitting
}

2

3 Felicity R Gill a, Paul J Murphy ${ }^{\mathrm{a}, \mathrm{b}}$ and Christine Purslow ${ }^{\mathrm{a}}$

4

$5 \quad{ }^{a}$ Cardiff University, School of Optometry and Vision Sciences, Cardiff, UK

6 b University of Waterloo, School of Optometry and Vision Science, Waterloo, Canada

8 Tables: 2

9 Figures: 7

10

11 Address for correspondence:

12 Prof Paul J Murphy

13 University of Waterloo

14 School of Optometry and Vision Science

15200 Columbia Avenue West

16 Waterloo, N2L 3G1

17 Ontario, Canada

18

19 Email: pjmurphy@uwaterloo.ca

\section{$22 \quad$ Funding}

23 This work was supported by an Industrial CASE-EPSRC scholarship from No7 Contact Lenses, Hastings, East 24 Sussex, UK, and the UK Engineering and Physical Sciences Research Council (EPSRC). 


\section{Abstract}

28 Purpose: To investigate effect of topical anaesthetic (TA) during gas permeable (GP) contact lens (CL) fitting 29 on subjective and objective measures of patient anxiety.

Methods: 47 subjects (mean \pm sd age $=26.9 \pm 4.9$ years; soft $C L$ wearers, 18 , neophytes, 29 ). Each subject randomly assigned to Group A or B, and attended on two occasions, one week apart. First visit: subject received bilaterally either a single drop of TA $(0.5 \%$ proxymetacaine) (Group A) or placebo ( $0.9 \%$ saline)

34 (Group B) prior to GP CL application. No drops were instilled at second visit. Each visit mimicked a GP CL 35 fitting. At each visit, patient anxiety was assessed either subjectively (visual analogue scale (VAS)) or objectively (skin conductance (SC)), as well as anterior ocular health.

Results: Visit 1: GP CL trial produced small increases in hyperaemia and corneal staining, but no difference associated with TA use. Visit 2: increases in staining and hyperaemia were observed, but hyperaemic responses significantly less than at Visit 1 , for both groups. Corneal staining also less, but not statistically significant. VAS scores indicated subjects who received TA during Visit 1 were significantly less anxious at Visit 2. Visit 2: comfort slightly reduced for subjects who received TA at Visit 1, and significantly increased for subjects who received placebo. Use of TA reduced anxiety during lens adaptation period compared with subjects receiving placebo.

Conclusions: TA use during GP CL fitting has potential patient benefits: improved first-time GP CL wear comfort, reduced anxiety during adaptation, reduced anxiety prior to subsequent GP CL wear.

Keywords: GP contact lenses, anxiety, anaesthesia, comfort, success

\section{Highlights}

Use of topical anaesthetic during gas permeable (GP) contact lens (CL) fitting is clinically safe, improves firsttime GP CL wear comfort, reduces anxiety during adaptation and in subsequent $\mathrm{CL}$ wear. 
The decline in rigid gas permeable (GP) contact lens $(C L)$ prescribing is well documented ${ }^{1}$. In a previous study, we showed that the initial wearing discomfort with GP CLs discourages practitioners from recommending this lens type to patients ${ }^{2}$. Topical anaesthetic (TA) use in rigid gas permeable fitting results in enhanced initial patient comfort ${ }^{3}$, and may also reduce patient anxiety about initial lens comfort ${ }^{3}$. If initial comfort is improved with TA, particularly in patients perceived to have high ocular touch sensitivity or are anxious, practitioners may feel encouraged to consider GP CLs as a potential option ${ }^{4}$. However, the use of topical anaesthetic to aid GP CL fitting, is not common practice in the United Kingdom and practitioner opinion is divided on the acceptability of TA during GP CL fitting without evidence on the safety and benefit of TA use.

Anxiety is the adaptive response to a threat, for example, in response to a clinical procedure ${ }^{5}$. Anxiety is known to influence patient success with $\mathrm{CL}^{6.7}$. It has been suggested that patients may not try $\mathrm{CL}$ because they are anxious about having them placed on their eyes ${ }^{7}$. Anxiety levels appear to vary between individuals and both internal and external forces may influence anxiety levels. Spielberger ${ }^{8}$ suggested that 'trait' anxiety refers to a person prone to anxiety, i.e. it is a fixed personality trait, while 'state' anxiety is a transient anxiety experience ${ }^{8}$.

Use of TA makes the first GP CL experience more comfortable, but this raises questions over whether this makes the next visit, without TA, a worse experience, and therefore misleads a patient. Literature shows that use of TA results in less patient dropouts following the fitting phase ${ }^{3}$, however an insight into patient experience over the fitting phase would be advantageous.

This study investigated the effects of TA use, during GP CL fitting, on the ocular surface to assess its safety of use; on subjective and objective measures of patient anxiety; and of previous TA use on the second patient experience with GP CL.

\section{Methods}

83 A prospective, randomised, double-masked cohort study was conducted involving two visits, scheduled with 84 one week between visits.

Subjects Forty-seven healthy, volunteer, subjects from staff and students within Cardiff University completed the study, $m$ 20, f 27, mean \pm sd age $=26.9 \pm 4.9$ years (range 18-45). Twenty-nine subjects were neophyte and 18 
had experience of or were current soft $\mathrm{CL}$ wearers. Subjects were excluded if they had worn GP CL before, suffered from any ocular condition including dry eye or any systemic condition known to affect the tear film or cornea, were taking any medication known to affect the tear film or cornea, or were pregnant or breastfeeding. Ethical permission for the study was obtained from the School of Optometry and Vision Sciences Ethical Committee and signed informed consent was obtained from all subjects. All procedures conformed to the tenets of the Declaration of the Helsinki.

Study Groups

Subjects were randomly assigned to two groups (A or B) and either received a single drop of TA (A) or saline placebo (B) prior to GP CL application at the first visit, in both eyes, respectively. Group A ( $n=25$ ) had a mean \pm sd age of $27.1 \pm 4.6$ years, $m$ 11, $f 14$. Group B ( $n=22)$ had a mean \pm sd age of $26.6 \pm 5.2$ years, $m 9, f 13$.

\section{State and trait anxiety questionnaire}

102 The Spielberger State-Trait Inventory (STAI) ${ }^{9}$ incorporates two 20-item question sets measuring state and 103 trait anxiety. The items are generic and the STAI has been used to measure anxiety in many healthcare 104 studies ${ }^{10,11,12}$. The full STAl is lengthy and has been shortened to two 6-item scales ${ }^{9,13,14}$. Each item has four 105 possible responses, with each response giving a score, and the anxiety result is found by summing the response scores. The shortened State-Trait scales were completed by all subjects prior to drop instillation and GP insertion.

\section{Visual analogue scale}

110 An anxiety visual analogue scale (VAS) was completed prior to GP CL application to indicate subject 111 anxiety $15,16,17$. Subjects were asked to mark their answer on the VAS to the question "How anxious do you

112 feel about having contact lenses on your eyes today?", between the two extremes of "Not at all anxious' and

113 'Very anxious'. A comfort VAS was completed after GP fitting to indicate how comfortable the lenses had 114 been on the eyes in response to the question "How did the contact lenses feel on your eyes today?", 115 between the two extremes of 'Not at all comfortable' and 'Very comfortable'.

\section{Skin conductance recording procedure}

118 Skin conductance (SC) shows the emotional state reflected by changes in the sympathetic nervous system as 119 a result of stress or arousal. Sympathetic activation causes release of acetylcholine, which acts on the muscarinic receptors leading to sweat production and a skin conductance increase ${ }^{18}$. SC has been used as a 
121 tool for monitoring post-operative pain in medicine ${ }^{19}$. It has been found to be better than alternative

122 objective methods, e.g. heart rate, blood pressure and electroencephalograph (EEG), at detecting pain ${ }^{18}$.

124 Skin conductance was measured by attaching 2 silver-silver chloride electrodes (coated with electrode gel) to 125 the pads of the index and middle finger of the subject's left hand. Signals from the electrodes were amplified $126(\mathrm{x} 2000)$ and low pass filtered $(0-35 \mathrm{~Hz})$ using a physiological amplifier (Biopac MP30) connected to a laptop

127 PC (Toshiba Satellite Pro 4200) running Biopac Student Lab Pro software (version 3.65, BIOPAC Systems Inc, 128 Goleta, CA). All subjects washed their hands with a liquid soap prior to having the electrodes attached to improve the quality of contact. A period of 10 minutes was allowed to elapse before data collection to ensure the skin had fully absorbed the gel. The subject was asked to keep their hand still, resting on their left

131 leg throughout the consultation. Conversation during the consultation was controlled and the same 132 explanations and reassurance were given to all participants.

SC response occurs with a latency of 1-3 seconds following a stimulus, making it difficult to directly link a response to a particular event ${ }^{23}$. For this reason, tags were helpful in marking periods of interest. Specific phrases were used by the examiner at key points during the consultation, and simultaneously the examiner added a tag to the trace (Figure 1). Tags were also added to the SC trace to identify completion of a particular task during the consultation. When subjects returned for the second visit, Tag 1 was omitted and only Tags 2-5 were inserted onto the SC trace.

140

Using the tags, information from the trace, such as mean response and maximal response, were determined within these periods of interest. Maximal response was selected as the key result for analysis in the results because this gave the subject's peak arousal or anxiety experienced within each period. Absolute SC values do not facilitate comparison of SC between individuals. Therefore, SC values recorded during the 'run-in period' (from the start of the trace until drop insertion) were averaged and subtracted from subsequent recordings to normalise the data in all subjects. 
155 At both visits, the health of the anterior eye was assessed using a slit-lamp. White light assessment allowed

156 grading of conjunctival and limbal hyperaemia, according to the CCLRU grading scale. A sodium fluorescein 157 sterile ophthalmic strip (FS) (Chauvin Pharmaceuticals, Romford, UK) was wetted with non-preserved $0.9 \%$ saline (Oxysept Saline; Abbott Medical Optics, High Wycombe, UK) and the FS applied to the inferior tarsal conjunctiva. Tear film fluorescence was enhanced with cobalt blue light, in conjunction with a Wratten Filter (No 12) in front of the objective lens. The corneal integrity was assessed and any corneal staining was recorded diagrammatically, and also graded using the CCLRU grading scale.

At the first visit only, corneal keratometry of both eyes was measured using a 2-position Javal-Schiötz type keratometer (Topcon Corporation, Tokyo, Japan).

\section{Schedule}

Subjects were invited to attend for two GP CL fitting sessions, with a one-week separation between the two visits. The first visit mimicked a first GP CL fitting session when either TA or placebo drops were instilled. The second visit mimicked a second GP CL fitting session and no drops were instilled.

Based on the keratometry measurement, an appropriate GP CL was selected from a fitting set (Quasar, No7 Contact Lenses, Hastings, UK). All lenses had a total diameter of $9.60 \mathrm{~mm}$ and back vertex power of -3.00 Dioptres. The required lenses were cleaned and rinsed using Boston Advance 2-step system (Bausch \& Lomb, Kingston-upon-Thames, UK). To mimick a CL fitting session, the selected CLs were then applied to both eyes while the patient's eyes were in down-gaze. Once the lenses were settled and tearing had reduced or stopped, the lens fit was assessed. The examiner advised the subject that lenses were to be removed, which was then done by placing mild pressure on the inferior and superior lid margins, and digitally moving the lids together to release the lens. Conjunctival hyperaemia and limbal injection were regraded and corneal staining was noted and graded. Further FS was instilled at this stage only if required, since successive FS instillation is known to increase corneal staining ${ }^{20}$.

182 Group A volunteers received 1 drop of $0.5 \%$ proxymetacaine hydrochloride (proparacaine) (Chauvin 183 Pharmaceuticals, Romford, UK) in both eyes. Group B received 1 drop of $0.9 \%$ saline (Chauvin Pharmaceuticals, Romford, UK) in both eyes. Coloured tape was used to code the minims to mask both subject and examiner to the drops being administered. 
On each visit, patient anterior ocular health, and subjective and objective patient anxiety were measured.

188 Keratometry was undertaken at the first visit only (Keratron Scout topographer (KS-1000), Optikon, Rome, 189 Italy).

\section{Statistical analysis}

192 Data was analysed using SPSS 16.0 (SPSS Inc., Chicago, USA) and examined for normality by the Shapiro-Wilk 193 test and appropriate statistical tests used. A probability value of $<0.05$ was used for statistical significance.

194 Differences between groups were assessed by unpaired t-test (parametric) or U-Test Mann-Whitney (nonparametric data). Internal reliability of the short version state and trait questionnaires was assessed using Cronbach alpha. Post-hoc Wilks' Lambda was used to assess within-group interactions. Interpolation of the CCLRU grading scale produces an approximate interval scale and it has been argued that parametrical statistical tests may be applied to such data ${ }^{20}$, consequently parametric tests have been used predominantly Statistically, no significant difference was found between the eyes and therefore right eye data is presented throughout.

201

\section{Results}

203 Physiological effects

204 At Visit 1, no significant difference was found in baseline ocular surface grading between the two groups

205 (Table 1). Following GP CL application, conjunctival and limbal hyperaemia, and corneal staining was 206 significantly increased in both groups when compared to their baseline measures (Figure 2). Comparison of 207 grading pre- and post-GP fitting revealed no significant differences between the groups for hyperaemia or corneal staining change. Likewise, comparison of final CCLRU scores revealed no statistical difference between the groups.

210

211 At Visit 2, no significant difference was found in baseline grades for limbal and conjunctival hyperaemia or 212 corneal staining between the groups. Following GP CL application, both groups showed an increase in mean 213 hyperaemia and corneal staining scores (Table 1) (Figure 3). There was a significant increase in hyperaemia

214 and corneal grading scores between the pre- and post-GP CL fitting for Group A. The hyperaemia increase in 215 Group B was not statistically significant when comparing before and after GP grading. Comparison of 216 difference in grade (pre- and post-GP) between groups revealed no significant difference in hyperaemic 217 response between the groups. Following GP CL fitting, corneal staining was significantly increased in both 218 groups, however there was a significantly greater corneal response in Group A compared with Group B 219 (Figure 4). 
221 Internal reliability of the short version state and trait questionnaires was assessed using Cronbach alpha.

222 Cronbach alpha values for state anxiety analysis were: Visit 1, $\alpha=0.97$; Visit 2, $\alpha=0.99$, indicating a high degree of consistency, and making comparison of state anxiety results statistically reliable ${ }^{22}$.

Inter-group trait scores were similar at Visit 1 and 2 for Group A ( $p=0.97$, Mann-Whitney) and Group B ( $p=0.63$, Mann-Whitney). State anxiety showed no significant difference between groups in baseline anxiety at Visit 1 ( $p=0.56$, Mann-Whitney). No significant change in state anxiety was evident between Visit 1 and 2 for Group A ( $p=0.35$, Mann-Whitney), but Group B had increased state anxiety at Visit $2(p<0.05$, MannWhitney) (Figure 5).

There was no significant difference between Group A and B VAS anxiety scores at Visit 1. At Visit 2, Group A were significantly less anxious about lens application. Group B were marginally more anxious at Visit 2, though this finding was not statistically significant. Comparison of the change in anxiety over the two visits, between groups, was not significant (Table 2) (Figure 6).

At Visit 1, initial GP VAS comfort scores were higher in Group A compared with Group B, but this difference was not statistically significant. At Visit 2, comfort scores significantly decreased in Group A and increased in Group B (Table 2) (Figure 7).

\section{Skin conductance}

For Visit 1, a mixed, between-within subjects ANOVA was conducted to assess the impact of two different interventions (effect of drops) on subjects' maximal SC response across three time periods (lens insertion, adaptation to lenses, and lens removal). There was no significant interaction between drop and time (Wilks Lambda, $p=0.97)$. There was no significant main effect for time $(p=0.97)$. The main effect comparing the groups, depending on the type of drop instilled, was not significant $(p=0.64)$. A one-way repeated measures ANOVA was conducted to compare maximal SC responses over time, but no significant effect of time was found (Group A, p=0.78; Group B, p=0.98).

For Visit 2, a mixed, between-within subjects ANOVA found no significant interaction between drop and time (Wilks Lambda, $p=0.82)$, nor was there a significant main effect for time $(p=0.84)$. The main effect comparing the groups, depending on the type of drop instilled, was not significant $(p=0.18)$. 


\section{Discussion}

254 The findings from this study indicate that TA is beneficial in reducing both objective anxiety measurements during adaptation to GP CLs and self-reported anxiety prior to second-time lens insertion, while producing no clinically significant physiological changes. However, this benefit for subsequent lens wear may produce a falsely raised expectation for future $\mathrm{CL}$ wearing comfort.

The use of TA during GP CL fitting has been demonstrated to be a clinically safe practice with potential patient benefits including improved first-time GP CL wear comfort, reduced anxiety during adaptation and reduced anxiety prior to second-time GP CL wear. The use of TA itself did not adversely increase ocular surface hyperaemia or corneal staining response during lens fitting. At the second visit, the ocular redness response to GP CLs was reduced, irrespective of previous drop experience (TA or placebo). Comfort at initial fitting was marginally improved with TA, although it was worse at the dispensing visit. Patients who received TA during fitting had significantly reduced anxiety (VAS) prior to lens collection, suggesting that this practice may minimise $\mathrm{CL}$ drop-out rates. The disadvantages of TA use may be the reduced comfort during secondtime GP CL wear when no TA is administered.

These findings concur with a previous study which reported reduced drop-out rates in first-time wearers fitted with use of TA at fitting and dispensing visits ${ }^{4}$. A similar study fitted apprehensive patients using TA and reported superior comfort, less alteration to blink rate and less tearing compared with a control group. Furthermore, $50 \%$ of subjects felt confident about wearing GP CLs following fitting with TA compared with $20 \%$ of control subjects ${ }^{4}$. The study also reported the use of TA to significantly reduce time for GP CL stabilisation on the eye. (GP CL stabilisation time, blink rate or lacrimation were not measured during this investigation). Effect of TA on GP CL stabilisation time might be of interest as the time needed to fit GP CLs is perceived to be greater than that for soft CL fitting. The use of TA to shorten fitting appointments might be a further indication for TA use in GP CL fitting.

Physiological response

280 The collective mean $(n=47)$ baseline bulbar conjunctiva hyperaemia CCLRU grade was $1.81 \pm 0.27$ units at Visit

2811 and $1.70 \pm 0.21$ units at Visit 2. Murphy et al (2007) indicated that bulbar conjunctiva hyperaemia grading 282 with the CCLRU normally ranges from 1.3-2.6 units, and a grade of more than 2.6 should be considered 283 abnormal. Most eye care practitioners (ECPs) would accept that slight increases in ocular surface hyperaemia 284 occur when CLs are first applied. Due to inter-subject variability, measurement of change in bulbar conjunctiva hyperaemia is more meaningful than absolute values, with a change of 0.4 units considered as 
clinically significant ${ }^{24}$. The results here indicate that the mean increase in hyperaemia grades during the GP

287 CL trial were small (less than one quarter of a CCLRU grade), but statistically significant. Importantly, the study demonstrated that use of TA did not promote a clinically significant increase in hyperaemia in this cohort.

291 Hyperaemia increase at Visit 2 was statistically more significant in Group A than Group B. A possible 292 explanation for these findings might be that the Group B hyperaemic reaction was conditioned by an 293 improvement in comfort experience at the second exposure to GP lenses. Meanwhile, subjects in Group A, who received TA at Visit 1 experienced a reduced level of ocular comfort at Visit 2, and therefore responded as if they were naïve to GP CLs. An alternative explanation might be that, while baseline hyperaemia grades were greater in Group B than Group A $(p=0.06)$, the mean increase in redness was small and similar $(p<0.05)$ for both groups.

It has been reported that a mean CCLRU corneal staining grade of 0.1 (max 0.5 ) should be anticipated for non- $\mathrm{CL}$ wearers ${ }^{25}$. However, the cohort reported here included both non-CL wearers and soft $\mathrm{CL}(\mathrm{SCL})$ wearers. SCL wear alters cell exfoliation and proliferation in the corneal and limbal epithelia resulting in increased staining ${ }^{26,27}$. This study found a mean baseline corneal staining grade of $0.23 \pm 0.39$ units, which was marginally higher than the Dundas et al. ${ }^{25}$ study for non-CL wearers, and marginally less than the mean Grade 0.5 reported in a study of asymptomatic hydrogel $C L$ wearers ${ }^{28}$. Our study found $<0.1$ unit difference in mean corneal staining grade (post-GP CL wear) between the placebo and TA group. Although mean change in corneal staining grade was larger in the TA group, this difference was not statistically significant. Similar studies have also reported no significant increase in corneal staining with TA use compared with a control drop ${ }^{29,30}$.

This result is perhaps surprising given that most optometrists will anecdotally report a reluctance to use TA due to its 'toxic effect'. Yet, UK practitioners routinely instil TA prior to clinical techniques such as Goldmann applanation tonometry ${ }^{24}$. Clinicians are aware of the potential risks associated with TA use, but consider that

313 the benefits of producing corneal anaesthesia outweigh them. Indeed, TA is known to be mildly toxic to the 314 corneal epithelium ${ }^{20}$. One study investigating corneal staining reported $17.6 \%$ of eyes stained with 315 fluorescein at baseline measurement, but that following TA instillation (oxybuprocaine and tetracaine), $60 \%$ 316 of eyes stained with fluorescein ${ }^{31}$. However, it is likely that the preservative $(0.01 \%$, benzalkonium chloride) 317 accompanying the TA in that study was responsible for the staining increase. Research has reported that 318 sequential instillation of TA was not responsible for increased epithelial permeability, but the addition of 
preservatives significantly increases corneal permeability ${ }^{31}$. Preservative-free TA minims $(0.5 \%$,

320 proxymetacaine) were used in this study to reduce the risk of ocular surface response associated with

321 preservative. Repeated use of TA can delay wound healing or cause keratitis ${ }^{31}$, but only one drop of TA was 322 used in this study.

323

324 At Visit 2, the results indicated that corneal staining was increased in all subjects following GP CL insertion, 325 but the mean grade increase was not clinically significant for either Group A or B ${ }^{34}$.

\section{Physiological response}

328 Measured trait anxiety at the start of each visit (although not expected to change between visits) confirmed 329 an even distribution of tendencies toward anxiety in both groups, i.e. there was no skew in either group 330 towards very sensitive individuals. State anxiety refers to the transient or current level of anxiety experienced by the subject. Variations in volunteer personality types and extraneous factors, which might have influenced state anxiety levels, may produce the wide variation observed in results prior to the Visit 1

$333 \mathrm{CL}$ trial. Importantly, both measures of anxiety (state anxiety and VAS) were not significantly different 334 between Groups A and B at Visit 1. Both groups were naïve to GP CLs and masked as to whether they would 335 receive TA or placebo drops.

At Visit 2, subjects who had previously received TA at Visit 1, showed less anxiety when measured with the VAS, but no significant change in state anxiety scoring. It may be that the state score was affected by extraneous stress factors and this masked the reduction in anxiety relating specifically to GP CL insertion. Conversely, the placebo group state anxiety scores showed a significant increase at Visit 2 implying that their negative experience at Visit 1 caused them to feel more anxious in anticipation of GP CL insertion for the second time. However, this was not the case for their anxiety VAS responses, which showed no significant change from Visit 1. This is perhaps because subjects were no longer naïve to GP CLs and knew what to expect (i.e. no fear of the unknown as at Visit 1). Social anxiety research indicates that within a formal encounter people generally want to make a good impression and want to avoid appearing foolish ${ }^{34}$. Therefore, an alternative explanation may be that subjects were too embarrassed to admit to feeling anxious at the prospect of second-time GP CL discomfort experience, a condition more easily expressed on a simple VAS. 
experienced better comfort, and consequently reduced stress levels. Apart from reduced corneal sensitivity,

353 other factors which may affect stress levels during adaptation to lenses might have included change in vision

354 due to the power of the trial lens (-3.00 Dioptres), acceptability of the CL fit, and individual lid architecture or tightness. However, the effects of these factors should have been equal for both groups.

At Visit 2, SC appeared somewhat heightened in the anaesthetic group because they now experienced the full sensation of the GP CL, whereas Group B had lower SC response since they experienced an improved level of comfort at second exposure to GP CLs. However, statistically there was no difference in the results for the two groups.

Electrodermal activity is the most widely accepted measure of arousal or anxiety, and SC is the best objective measurement of electrodermal activity ${ }^{36}$. Previous research has investigated SC during soft CL fitting and reported characteristic anxiety fluctuations during the consultation. Specifically, heightened stress response during $\mathrm{CL}$ insertion and $\mathrm{CL}$ removal was reported ${ }^{37,38}$. Visual inspection of each trace produced by subjects in this study found heightened SC response during CL insertion and removal. However, this research was specifically interested in alterations to the SC response due to the use of TA during GP CL fitting. The trends shown in the results indicate that there may be a reduction in anxiety with $\mathrm{TA}$, however the results were not statistically significant. Trends may become significant with increased sample size.

370

At Visit 2, VAS comfort levels were improved in the group (A) that received TA prior to initial CL fitting, but this was not significantly better than the placebo group (B) $(p=0.12)$. This lack of statistical significance may be because there was a wide variation in comfort scores and the sample size. If the cohort had been larger, it is likely that this trend would have shown statistical significance. It may be that the superior palpebral conjunctiva is less well anaesthetised due to application of the drop to the inferior palpebral conjunctiva. This is supported by the idea that comfort during GP CL wear may be more directly linked to sensitivity of the superior tarsal plate and the position of the $\mathrm{CL}$ margin in relation to the superior lid $^{3}$.

378

379 It is possible that there could be a negative outcome from the use of TA, arising from the decreased comfort experienced by first visit TA subjects, at the second non-TA visit. In this situation, the subject experiences more discomfort, which may promote cessation of GP CL wear. However, the reduced anxiety levels at the second visit for the first visit TA subjects is a strong indication that subjects, although experiencing higher levels of discomfort, are calmer about the whole lens fit process. This tends to support the benefit of TA use 
385 would be a useful extension of this study, by allowing the investigation of whether lens fit complexity can 386 have an impact on patient anxiety.

387

388 In summary, use of TA in GP CL fitting has been demonstrated to be clinically safe practice that may enhance

389 first GP CL lens experience, especially in anxious patients, reduce anxiety during GP CL adaptation, and 390 reduce anxiety prior to subsequent GP CL wear.

391

392 
394 1. Morgan PB, Woods CA, Tranoudis IG et al. International contact lens prescribing in 2015. Contact Lens Spectrum; Jan 2016. http://www.clspectrum.com/issues/2016/january-2016/international-contact-lensprescribing-in-2015 (accessed 20.01.17).

2. Gill FP, Murphy PJ, Purslow C. A survey of UK practitioner attitudes to the fitting of rigid gas permeable lenses. Ophthal. Physiol. Opt. 30 (2010) 731-739.

3. Bennett ES, Smythe J, Henry VA, Bassi CJ, Morgan BW, Miller W, Jeandervin M, Henderson B, Elliott L, Porter KS Barr JT. Effect of topical anesthetic use on initial patient satisfaction and overall success with

4. Schnider C. Anesthetics and RGPs: crossing the controversial line. Rev. Optometry 41 (1996) 41-43.

5. Shute R. It's worse than going to the dentist. Optom. Manag. October (1986) 10-12.

6. Hewett TT. A survey of contact lens wearers. Part II: Behaviors, experiences, attitudes, and expectations. Am. J. Optom. Physiol. Opt. 61 (1984) 73-79.

7. Hutchison G. Consumer and practitioner attitudes to contact lenses. Optician (2001) 17-21.

8. Spielberger C, Smith LH. Anxiety (drive), stress, and serial-position effects in serial-verbal learning. J. Exp. Psych. 72 (1966) 589-595.

9. Spielberger C, Gorsuch RL, Lushene R. Manual for the state/trait anxiety inventory. Consulting Psychologists Press, Palo Alto, 1983.

10. Cruise CJ, Chung F, Yogendran S, Little D. Music increases satisfaction in elderly outpatients undergoing cataract surgery. Can. J. Anaesth. 44 (1997) 43-48.

11. Farmer AJ, Doll H, Levy JC, Salkovskis PM. The impact of screening for Type 2 diabetes in siblings of patients with established diabetes. Diab. Med. 20 (2003) 996-1004.

12. Sari Z, Uysal T, Karaman Al, Sargin N, Ure O. Does orthodontic treatment affect patients' and parents' anxiety levels? Eur. J. Orthod. 27 (2005) 155-159.

13. Marteau TM, Bekker H. The development of a six-item short-form of the state scale of the Spielberger State-Trait Anxiety Inventory (STAI). Br. J. Clin. Psychol. 31 (1992) 301-306.

14. Court H, Greenland K, Margrain TH. Predicting state anxiety in optometric practice. Optom. Vis. Sci. 86 (2009) 1295-1302.

15. Wewers ME, Lowe NK. A critical review of visual analogue scales in the measurement of clinical phenomena. Res. Nurs. Health 13 (1990) 227-236.

16. Price DD, McGrath PA, Rafii A, Buckingham B. The validation of visual analogue scales as ratio scale measures for chronic and experimental pain. Pain 17 (1983) 45-56. 
17. Fujita H, Sano K, Sasaki S, Ohno-Matsui K, Tanaka T, Baba T, Mochizuki M. Ocular discomfort at the initial wearing of rigid gas permeable contact lenses. Jpn. J. Ophthalmol. 48 (2004) 376-379.

18. Storm H. Changes in skin conductance as a tool to monitor nociceptive stimulation and pain. Curr. Opin. Anaesthesiol. 21 (2008) 796-804.

19. Ledowski T, Bromilow J, Wu J, Paech MJ, Storm H, Schug SA. The assessment of postoperative pain by monitoring skin conductance: results of a prospective study. Anaesthesia 62 (2007) 989-993.

20. Josephson JE, Caffery BE. Corneal staining after instillation of topical anesthetic (SSII). Invest. Ophthalmol. Vis. Sci. 29 (1988) 1096-1099.

21. Barbeito R, Simpson TL. Should level of measurement considerations affect the choice of statistic? Optom. Vis. Sci. 68 (1991) 236-242.

22. Bland JM, Altman DG. Statistics notes: Cronbach's alpha. BMJ 314 (1997) 572.

23. Dawson M, Schell A, Filion D. The electrodermal system. In: Cacioppo JT, Tassinary LG, Bernston GG (Eds.), Handbook of Psychophysiology. Cambridge University Press, Cambridge, 2000, pp. 200-203.

24. Murphy PJ, Lau JSC, Sim MML, Woods RL. How red is a white eye? Clinical grading of normal conjunctival hyperaemia. Eye 21 (2007) 633-638.

25. Dundas M, Walker A, Woods RL. Clinical grading of corneal staining of non-contact lens wearers. Ophthal. Physiol. Opt. 21 (2001) 30-35.

26. Ren DH, Petroll WM, Jester JV, Ho-Fan J, Cavanagh HD. The relationship between contact lens oxygen permeability and binding of Pseudomonas aeruginosa to human corneal epithelial cells after overnight and extended wear. CLAO J. 25 (1999) 80-100.

27. Ladage PM, Yamamoto K, Ren DH, Li L, Jester JV, Petroll WM, Bergmanson JP, Cavanagh HD. Proliferation rate of rabbit corneal epithelium during overnight rigid contact lens wear. Invest. Ophthalmol. Vis. Sci. 42 (2001) 2804-2812.

28. Begley CG, Barr JT, Edrington TB, Long WD, Mckenney CD, Chalmers RL. Characteristics of corneal staining in hydrogel contact lens wearers. Optom. Vis. Sci. 73 (1996) 193-200.

29. Sturrock JE, Nunn JF. Cytotoxic effects of procaine, lignocaine and bupivacaine. Br. J. Anaesth. 51 (1979) 273-281.

30. Boljka M, Kolar G, Vidensek J. Toxic side effects of local anaesthetics on the human cornea. Br. J. Ophthalmol. 78 (1994) 386-389.

31. Ramselaar JA, Boot JP, van Haeringen NJ, van Best JA, Oosterhuis JA. Corneal epithelial permeability after instillation of ophthalmic solutions containing local anaesthetics and preservatives. Curr. Eye Res. 7 (1988) 947-950. 
32. Rosenwasser GO, Holland S, Pflugfelder SC, Lugo M, Heidemann DG, Culbertson WW, Kattan H. Topical anesthetic abuse. Ophthalmology 97 (1990) 967-972.

459 33. Lawrenson JG, Edgar DF, Tanna GK, Gudgeon AC. Comparison of the tolerability and efficacy of unitdose, preservative-free topical ocular anaesthetics. Ophthal. Physiol. Opt. 18 (1988) 393-400.

461 34. Schlenker BR, Leary MR. Social anxiety and self-presentation: a conceptualization and model. Psychol. 462 Bull. 92 (1982) 641-669.

463 35. Margrain TH, Greenland K, Anderson J. Evaluating anxiety in patients attending optometric practice. $464 \quad$ Ophthal. Physiol. Opt. 23 (2003) 287-293.

36. Court H, Greenland K, Margrain TH. Evaluating patient anxiety levels during contact lens fitting. Optom. 466 Vis. Sci. 85 (2008) 574-580.

467 37. Carney LG, Mainstone JC, Carkeet A, Quinn TG, Hill RM. Rigid lens dynamics: lid effects. CLAO J. 23 (1997) 69-77.

38. Bennett ES. Improving patient success with RGPs. Optometry Today July $30^{\text {th }}(1990) 42-44$.

470 


\begin{tabular}{|c|c|c|c|c|c|}
\hline \multicolumn{2}{|c|}{ Visit 1} & \multirow{2}{*}{$\begin{array}{l}\text { Pre-GP } \\
\text { fitting } \\
\text { Mean£sd }\end{array}$} & \multirow{2}{*}{$\begin{array}{l}\text { Post-GP } \\
\text { fitting } \\
\text { Mean } \pm \text { sd }\end{array}$} & \multirow{2}{*}{$\begin{array}{c}\text { Difference in } \\
\text { grading Pre- and } \\
\text { Post-GP wear } \\
\text { Mean } \pm s d \\
\text { (Paired t-test) }\end{array}$} & \multirow{2}{*}{$\begin{array}{c}\text { Difference } \\
\text { between Groups } \\
\text { A and B } \\
\text { Mean } \pm \text { sd } \\
\text { (Ind t-test) }\end{array}$} \\
\hline & & & & & \\
\hline \multirow{2}{*}{$\begin{array}{l}\text { Conjunctival } \\
\text { hyperaemia }\end{array}$} & Group A & $1.84 \pm 0.28$ & $2.08 \pm 0.43$ & $0.25 \pm 0.25(p<0.05)$ & \multirow{2}{*}{$\begin{array}{c}0.10 \pm 0.64 \\
(p=0.15)\end{array}$} \\
\hline & Group B & $1.78 \pm 0.26$ & $1.93 \pm 0.34$ & $0.15 \pm 0.16(p<0.05)$ & \\
\hline \multirow{2}{*}{$\begin{array}{c}\text { Limbal } \\
\text { hyperaemia }\end{array}$} & Group A & $1.59 \pm 0.42$ & $1.91 \pm 0.50$ & $0.26 \pm 0.56(p<0.05)$ & \multirow{2}{*}{$\begin{array}{c}0.01 \pm 0.14 \\
(p=0.93)\end{array}$} \\
\hline & Group B & $1.52 \pm 0.28$ & $1.78 \pm 0.40$ & $0.27 \pm 0.26(p<0.05)$ & \\
\hline \multirow{2}{*}{$\begin{array}{l}\text { Corneal } \\
\text { staining }\end{array}$} & Group A & $0.19 \pm 0.27$ & $0.63 \pm 0.66$ & $0.44 \pm 0.56(p<0.05)$ & \multirow{2}{*}{$\begin{array}{c}0.17 \pm 0.16 \\
(p=0.30)\end{array}$} \\
\hline & Group B & $0.28 \pm 0.49$ & $0.55 \pm 0.66$ & $0.27 \pm 0.54(p<0.05)$ & \\
\hline \multirow{2}{*}{\multicolumn{2}{|c|}{ Visit 2}} & $\begin{array}{l}\text { Pre-GP } \\
\text { fitting }\end{array}$ & $\begin{array}{l}\text { Post-GP } \\
\text { fitting }\end{array}$ & $\begin{array}{c}\text { Difference in } \\
\text { grading Pre- and } \\
\text { Post-GP wear }\end{array}$ & $\begin{array}{c}\text { Difference } \\
\text { between Groups } \\
\text { A and B }\end{array}$ \\
\hline & & Mean \pm sd & Mean \pm sd & $\begin{array}{c}\text { Mean } \pm s d \\
\text { (Paired t-test) }\end{array}$ & $\begin{array}{l}\text { Meanısd } \\
\text { (Ind t-test) }\end{array}$ \\
\hline \multirow{2}{*}{$\begin{array}{l}\text { Conjunctival } \\
\text { hyperaemia }\end{array}$} & Group A & $1.67 \pm 0.15$ & $1.78 \pm 0.21$ & $0.03 \pm 0.36(p<0.05)$ & \multirow{2}{*}{$\begin{array}{l}0.01 \pm 0.08 \\
(p=0.86)\end{array}$} \\
\hline & Group B & $1.73 \pm 0.27$ & $1.78 \pm 0.31$ & $0.04 \pm 0.12(p=0.11)$ & \\
\hline \multirow{2}{*}{$\begin{array}{c}\text { Limbal } \\
\text { hyperaemia }\end{array}$} & Group A & $1.51 \pm 0.34$ & $1.69 \pm 0.26$ & $0.10 \pm 0.42(p<0.05)$ & \multirow{2}{*}{$\begin{array}{c}0.03 \pm 0.10 \\
(p=0.73)\end{array}$} \\
\hline & Group B & $1.51 \pm 0.29$ & $1.56 \pm 0.31$ & $0.07 \pm 0.16(p=0.15)$ & \\
\hline \multirow{2}{*}{$\begin{array}{l}\text { Corneal } \\
\text { staining }\end{array}$} & Group A & $0.31 \pm 0.32$ & $0.68 \pm 0.49$ & $0.37 \pm 0.37(p<0.05)$ & \multirow{2}{*}{$\begin{array}{c}0.25 \pm 0.09 \\
(p<0.05)\end{array}$} \\
\hline & Group B & $0.32 \pm 0.41$ & $0.44 \pm 0.44$ & $0.12 \pm 0.17(p<0.05)$ & \\
\hline
\end{tabular}

474 Table 1: CCLRU grading measurements for Group A (TA) and Group B (placebo) at each visit.

475 
477

\begin{tabular}{|c|c|c|c|c|}
\hline \multicolumn{2}{|c|}{ Anxiety VAS } & $\begin{array}{l}\text { Group A } \\
\text { (TA drop) }\end{array}$ & $\begin{array}{l}\text { Group B } \\
\text { (placebo) }\end{array}$ & $\begin{array}{c}\text { Mann-Whitney } \\
\text { Test }\end{array}$ \\
\hline $\begin{array}{c}\text { Visit } 1 \text { score } \\
\text { (\%) }\end{array}$ & $\begin{array}{l}\text { Median } \\
\text { Range }\end{array}$ & $\begin{array}{c}13.57 \\
0.00-84.29\end{array}$ & $\begin{array}{c}9.29 \\
0.00-74.29\end{array}$ & $p=0.33$ \\
\hline $\begin{array}{c}\text { Visit } 2 \text { score } \\
\text { (\%) }\end{array}$ & $\begin{array}{l}\text { Median } \\
\text { Range }\end{array}$ & $\begin{array}{c}10.71 \\
0.00-35.71\end{array}$ & $\begin{array}{c}17.14 \\
0.00-55.71\end{array}$ & $p=0.31$ \\
\hline \multicolumn{2}{|c|}{ Wilcoxon Rank test } & $p<0.05$ & $p=0.94$ & \\
\hline \multicolumn{2}{|c|}{ Comfort VAS } & $\begin{array}{l}\text { Group A } \\
\text { (TA drop) }\end{array}$ & $\begin{array}{l}\text { Group B } \\
\text { (placebo) }\end{array}$ & $\begin{array}{c}\text { Mann-Whitney } \\
\text { Test }\end{array}$ \\
\hline $\begin{array}{c}\text { Visit } 1 \text { score } \\
\text { (\%) }\end{array}$ & Median Range & $\begin{array}{c}28.57 \\
2.86-100.00\end{array}$ & $\begin{array}{c}26.79 \\
2.86-97.86\end{array}$ & $p=0.25$ \\
\hline $\begin{array}{c}\text { Visit } 2 \text { score } \\
\text { (\%) }\end{array}$ & $\begin{array}{l}\text { Median } \\
\text { Range }\end{array}$ & $\begin{array}{c}22.86 \\
0.00-100.00\end{array}$ & $\begin{array}{c}58.57 \\
0.00-98.57\end{array}$ & $p=0.12$ \\
\hline \multicolumn{2}{|c|}{ Wilcoxon Rank test } & $p<0.05$ & $p<0.05$ & \\
\hline
\end{tabular}

478

479 Table 2: VAS anxiety and comfort results for Groups A and B at each visit.

480

481 
482 Figures

\section{Figure Legends}

484 Table 1: CCLRU grading measurements for Group A (TA) and Group B (placebo) at each visit.

485 Table 2: VAS anxiety and comfort results for Groups A and B at each visit.

486

487 Figure 1: Example of raw skin conductance trace, showing marker tags.

488 Figure 2: Error plots showing mean \pm sd in CCLRU grading scores pre- and post-GP fitting at Visit 1. A:

489 Conjunctival hyperaemia, B: Limbal hyperaemia and C: Corneal staining.

490 Figure 3: Error plots showing mean $\pm s d$ CCLRU grading scores pre- and post-GP fitting at Visit 2; A:

491 Conjunctival hyperaemia, B: Limbal hyperaemia and C: Corneal staining.

492 Figure 4: Mean change in CCLRU grading scores during Visits 1 and 2 for Groups A and B.

493 Figure 5: Box plot of median and range of state anxiety scores for Groups A and B at Visits 1 and 2.

494 Figure 6: Box plot of median and range of VAS anxiety scores for Groups A and B prior to GP insertion at 495 Visits 1 and 2.

496 Figure 7: Box plot of median and range of VAS comfort scores for Groups A and B at Visits 1 and 2. 
499

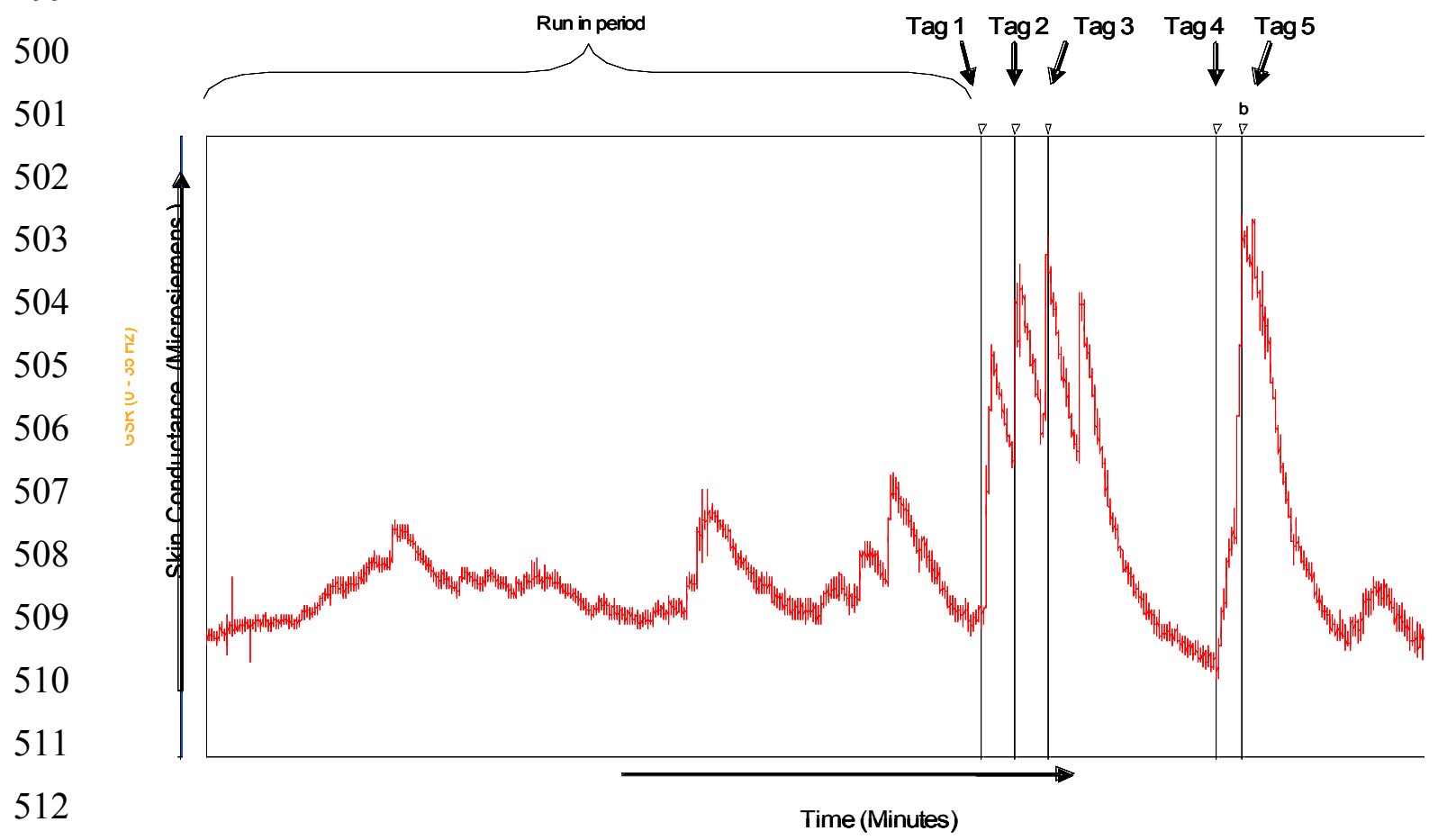

513 Figure 1: Example of raw skin conductance trace, showing marker tags. Tag 1: Examiner says: “I'm going to

514 out a drop into yours eyes now"; Tag 2: Examiner says: "I'm now going to insert the lenses to your eyes"; Tag

515 3: Completion of lens insertion; Tag 4: Examiner says: "I'm now going to remove the lenses from your eyes";

516 Tag 5: Completion of lens removal.

517 

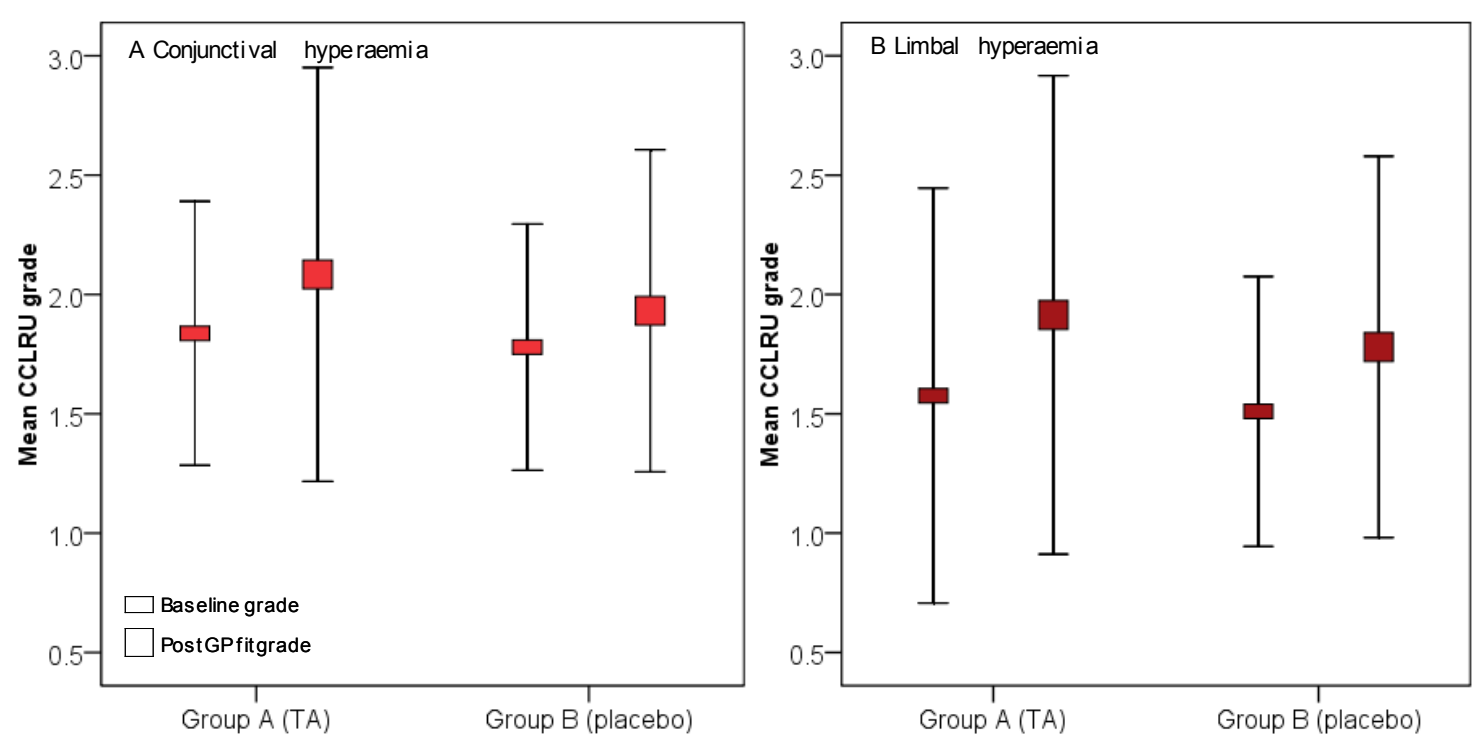

540 Figure 2: Error plots showing mean \pm sd CCLRU grading scores pre- and post-GP lens fitting at Visit 1. A:

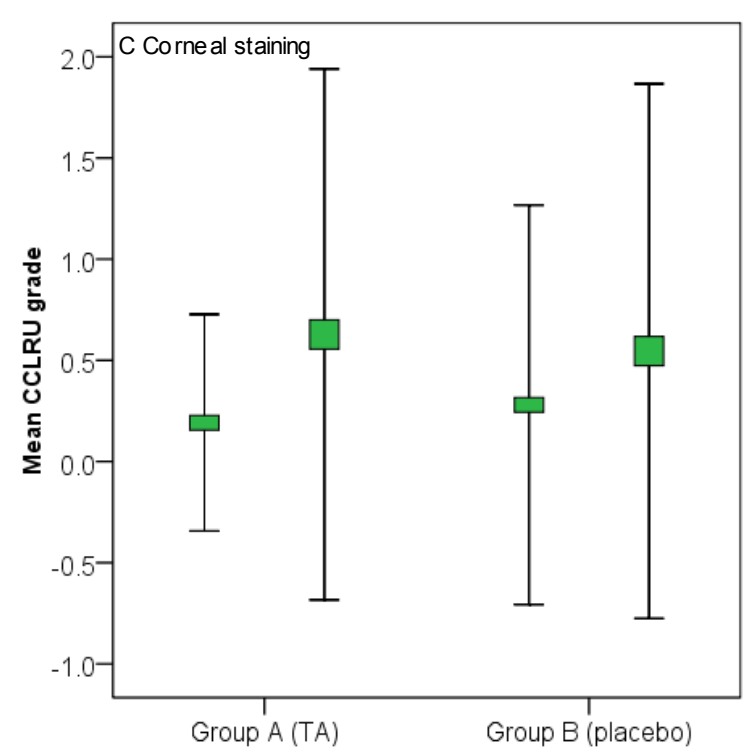

541 Conjunctival hyperaemia, B: Limbal hyperaemia and C: Corneal staining.

542 
544

545

546

547

548

549

550

551

552

553

554

555

556

557

558

559

560

561

562

563

564

565
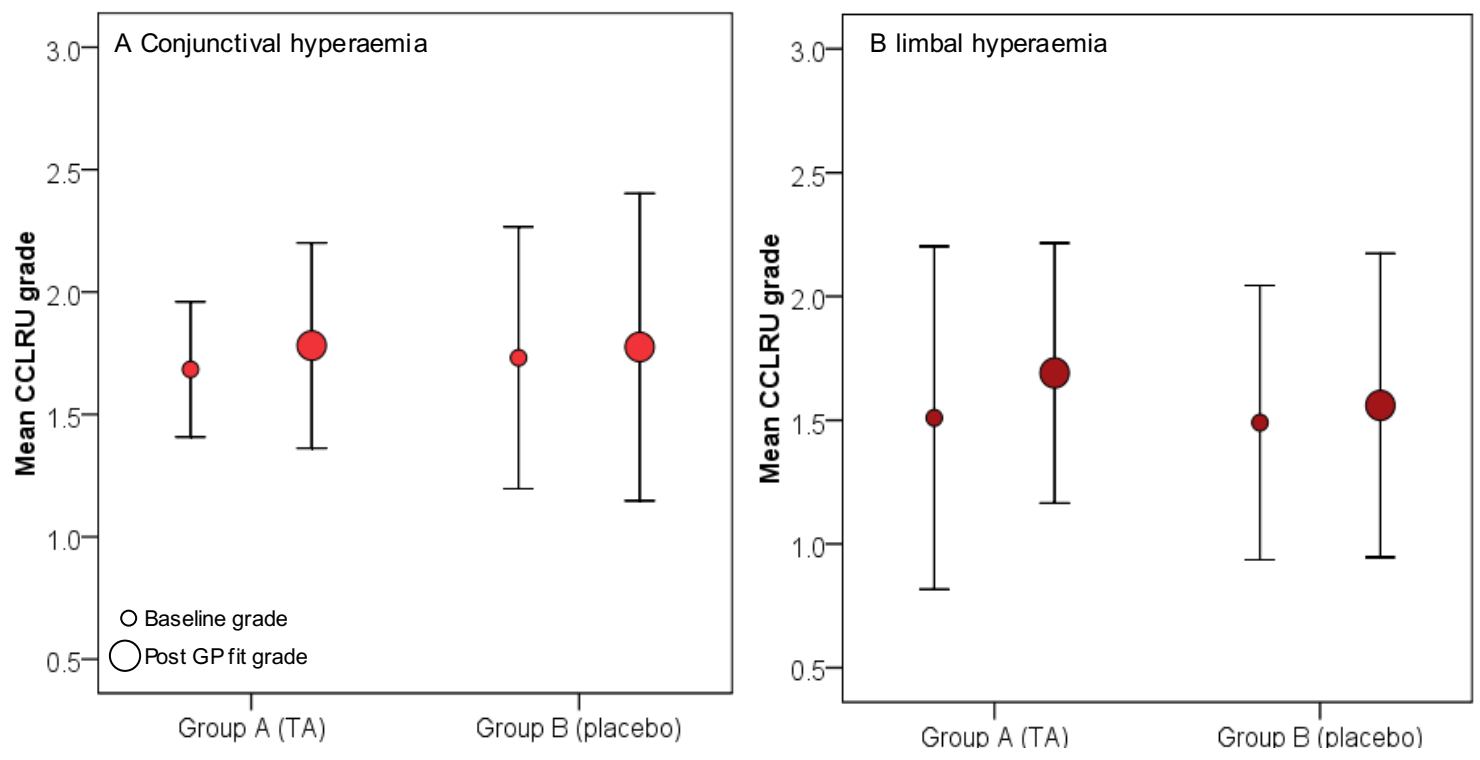

566

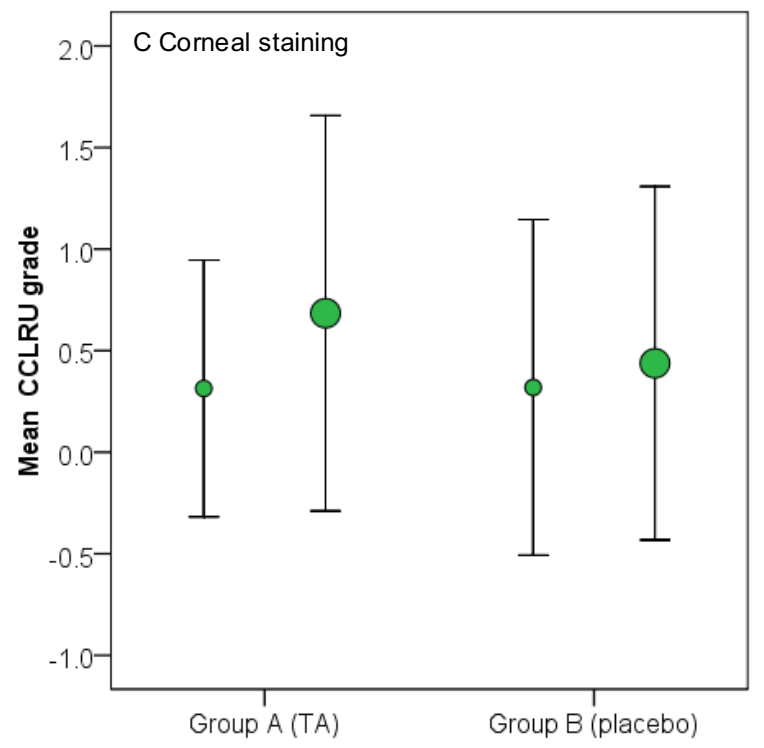

567 Figure 3: Error plots showing mean \pm sd CCLRU grading scores pre- and post-GP lens fitting at Visit 2; A:

568 Conjunctival hyperaemia, B: Limbal hyperaemia and C: Corneal staining.

569

570 


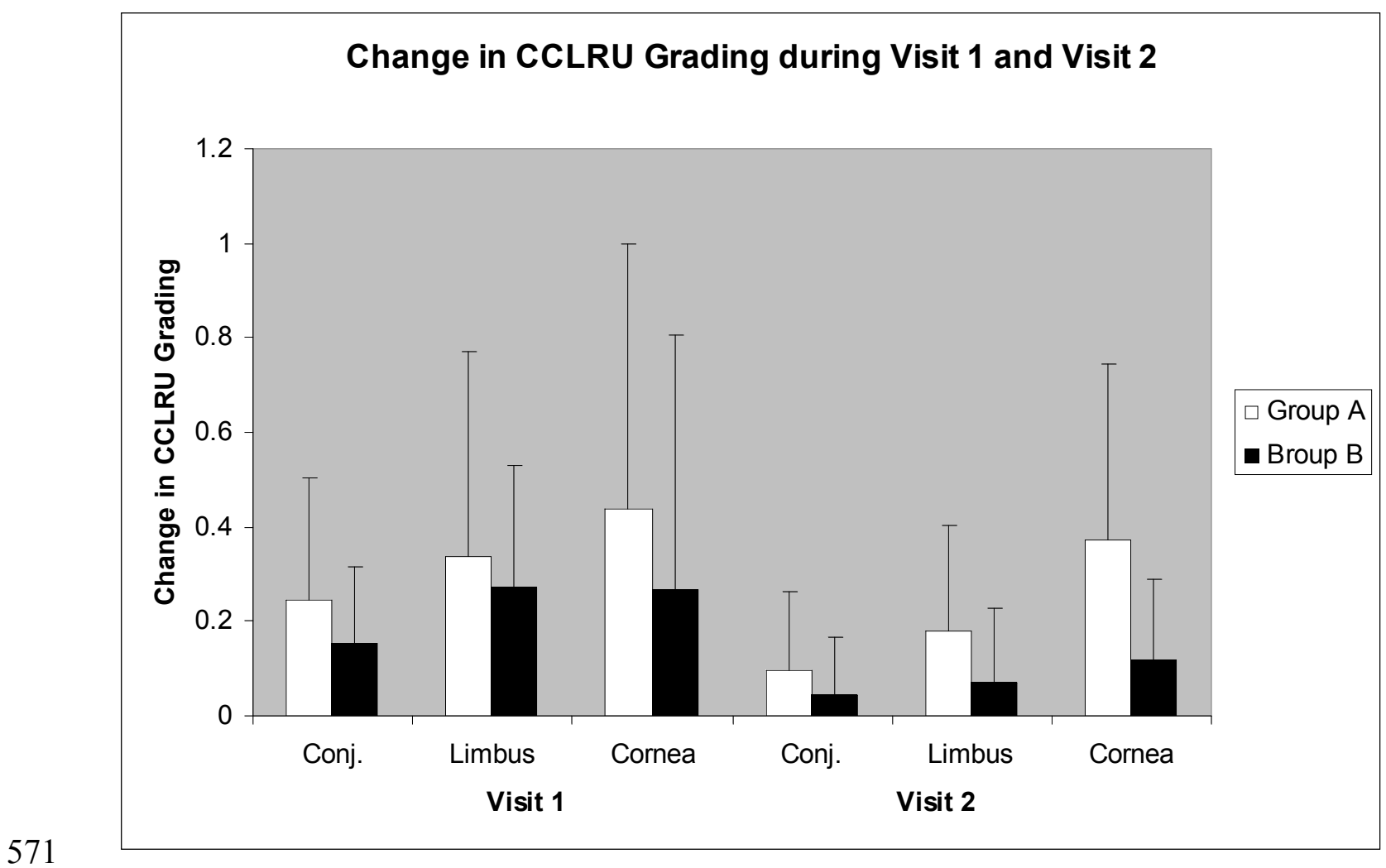

57

572 Figure 4: Mean change in CCLRU grading scores during Visits 1 and 2 for Groups A and B.

573 
575

576

577

578

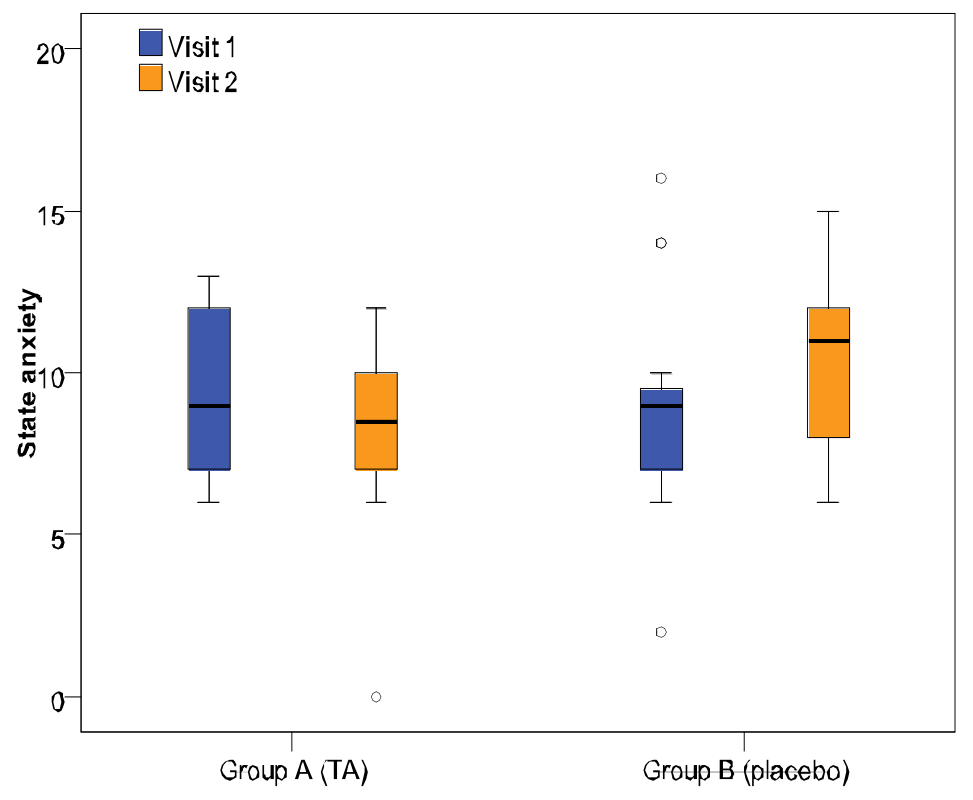

579 Figure 5: Box plot of median and range of state anxiety scores (from the shortened 6-item version of the 580 Spielberger State-Trait Inventory (STAI) questionnaire) for Groups A and B at Visits 1 and 2 (whiskers 581 represent the $10^{\text {th }}$ and $90^{\text {th }}$ percentiles).

582 
583

584

585

586

587

588

589

590

591

592

593

594

595

596

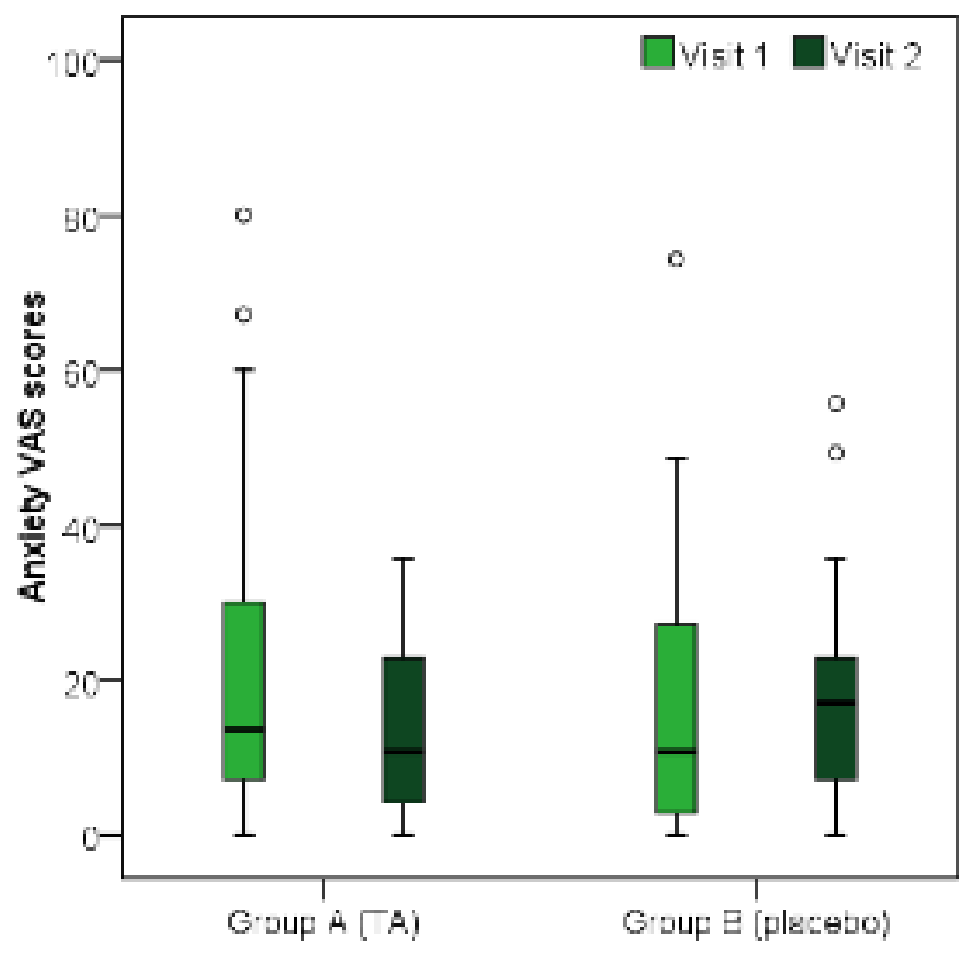

597 Figure 6: Box plot of median and range of VAS anxiety scores for Groups A and B prior to GP lens insertion at 598 Visits 1 and 2.

599 
600

601

602

603

604

605

606

607

608

609

610

611

612

613

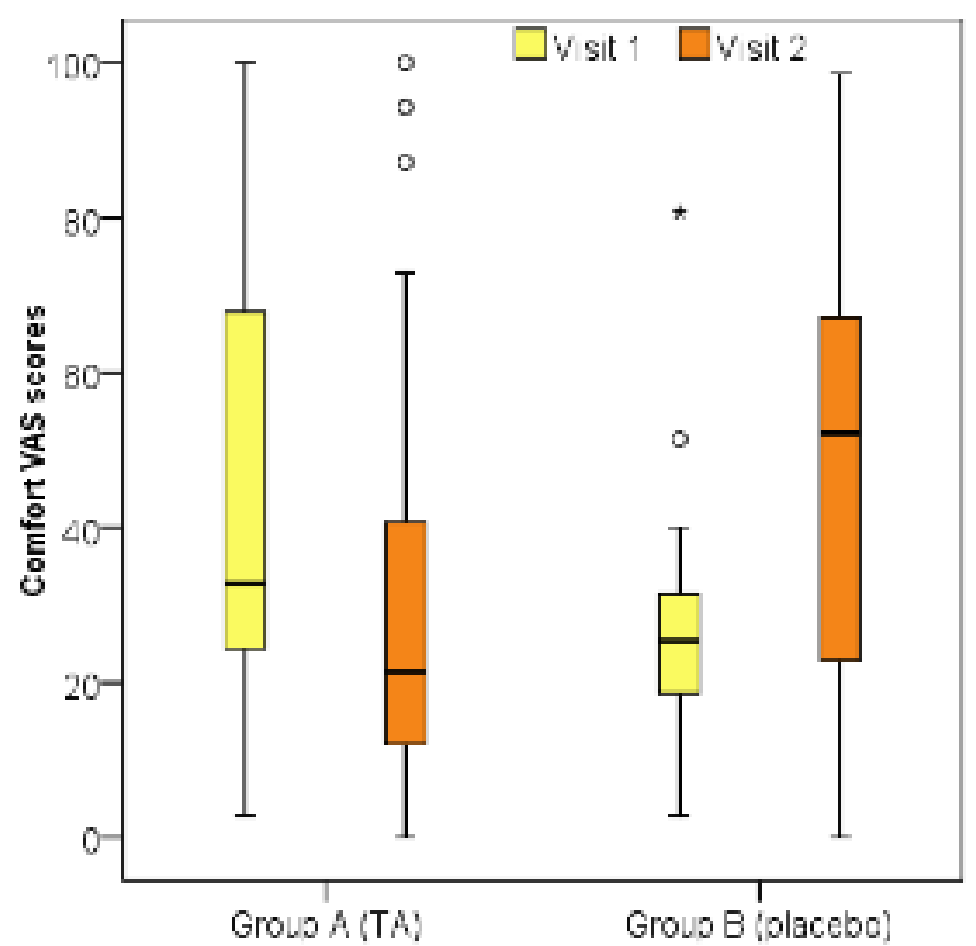

614 Figure 7: Box plot of median and range of VAS comfort scores for Groups A and B at Visits 1 and 2. 615 